\title{
X-ray Flare Rate of T Tauri Stars in Taurus
}

\author{
R. Neuhäuser ${ }^{1}$,Th. Preibisch ${ }^{2}$
}

1 Max-Planck-Institut für Extraterrestrische Physik, 85740 Garching, Germany

2 Universität Würzburg, Astronomisches Institut, Am Hubland, 97074 Würzburg, Germany

\begin{abstract}
We study the X-ray emission of several hundred (young, low-mass, late-type, pre-main sequence) T Tauri stars (TTS) in the Taurus T association, a nearby well-studied region of ongoing star formation. We report on X-ray emission variability of TTS as observed with the flux-limited ROSAT All-Sky Survey (RASS). Since RASS observations are spatially unbiased, we can investigate the $\mathrm{X}$-ray flare rate of TTS on a large sample. We find that large flares are very rare (once per year), while medium-size flares can occur once in $\sim 40$ days.
\end{abstract}

\section{Introduction}

TTS are fast rotators with periods down to a few days (e.g. Bouvier et al. 1993). A strong correlation between $X$-ray surface flux and stellar rotation first observed by Bouvier (1990) indicates that weak-emission line TTS (WTTS) are intrinsically more X-ray active than classical TTS (CTTS), because WTTS rotate faster. The rapid stellar rotation sustains the internal magnetic field. A dynamo effect produces $\mathrm{X}$-ray flares, and magnetic field lines emerge out of the stellar surface containing hot X-ray emitting plasma. TTS X-ray emission has first been observed with the Einstein Observatory. As expected from the model given above, irregular variability as well giant flares have been found in the X-ray light curves of TTS (see Montmerle et al. 1983).

\section{ROSAT observations}

As reported in Neuhäuser et al. (1995), CTTS are rarely (11\%) found with RASS, while most WTTS $(66 \%)$ are detected as strong X-ray sources; TTS Xray emission can be modelled with one-temperature Raymond-Smith (Raymond \& Smith 1977) spectra with energy peaking at $\sim 1 \mathrm{keV}\left(\sim 10^{7} \mathrm{~K}\right)$; WTTS and CTTS luminosity functions are significantly different; X-ray luminosity and surface flux are correlated with rotation period. Also, RASS observations confirm the irregular variability of TTS X-ray emission. However, due to low exposure time and, hence, statistical errors in RASS X-ray count rates, light curves are in many cases also not inconsistent with constant $\mathrm{X}$-ray emission. 
The X-ray emission model outlined above also predicts large flares. Preibisch et al. (1993) summarize properties of $\mathrm{X}$-ray flares and report on the largest such flare detected to date among TTS, the X-ray flare of $\mathrm{LH} \alpha 92$, observed during a ROSAT pointing on the Perseus $\mathrm{OB}$ association. The $\mathrm{X}$-ray telescope ROSAT has surveyed the sky in great circles, i.e., each object has been observed once in 90 minutes for up to $\sim 30$ seconds. In Taurus-Auriga, each TTS has been observed on average for 575 seconds, on average in 27 scans of average exposure time of 21 seconds. As typical TTS X-ray flares last several hours (e.g. Preibisch et al. 1993), i.e., longer than the separation between any two scans, RASS can detect any sufficiently large flare during RASS patrol time (start of first until end of last scan). The average RASS patrol time for a TTS in Taurus-Auriga is 41 hours.

In evaluating the $\mathrm{X}$-ray light curves, so far we have recognized flares just by visual inspection, accepting as flares those events with short rise time, e-folding decay time of several hours, and peak count rate significantly above quiescent level.

\section{Results}

We have detected one large X-ray flare on the single WTTS $040234+2143$. Also, we have found eleven medium-size flares including three peaks in GV Tau. In total, we have detected twelve flares among 256 TTS observed (including both TTS known prior to the ROSAT mission and newly discovered TTS). Eleven medium-size flares in 256 TTS observed (on average for 41 hours) correspond to roughly one flare in $\mathbf{4 0}$ days. The fact that we have observed only one large flare during this patrol time means that such large flares are very rare (once per year). These flare rates are very rough estimates and need to be improved by implementing objective flare recognition techniques on a larger sample including TTS in other star forming regions. Extending the X-ray flare luminosity versus flare rate plot (Montmerle et al. 1983) down to solar-type small and frequent flares can help distinguish whether TTS X-ray emission consists of many small flares or whether there is a truly quiescent level.

\section{References}

Bouvier J., 1990, AJ 99, 946

Bouvier J., Cabrit S., Fernandez M., Martin E.L., Matthews J.M., 1993, A\&A 272, 176

Montmerle T., Koch-Miramond L., Falgarone E., Grindlay J.E., 1983, ApJ 269, 182

Neuhäuser R., Sterzik M.F., Schmitt J.H.M.M., Wichmann R., Krautter J., 1995, A\&A (in press)

Preibisch Th., Zinnecker H., Schmitt J.H.M.M., 1993, A\&A 279, L33

Raymond J.C., Smith B.S., 1977, ApJS 35, 419 